tool to distinguish ACPA-negative elderly RA patients from PMR patients at initial presentation.

Methods: From April 2011 to December 2016, 21 RA patients and 24 PMR patients in our hospital, whose onset age was over 75 years, were recruited for this study. PMR patients did not have any evidence of giant cell arteritis. The diagnosis of RA was made based on 2010 ACR/EULAR RA classification criteria. The diagnosis of PMR was made based on 2012 EULAR/ACR classification criteria or Bird's criteria. Data were obtained from medical records under informed consent. Statistical analysis was performed using the Mann-Whitney U-test to compare median values and Fisher's exact test to compare frequencies (IBM SPSS version 24). $P<0.05$ indicated statistical significance.

Results: RA patients (6 men and 15 women) consisted of fifteen ACPA+ (11 RF+, $4 \mathrm{RF}-$ ), six ACPA- (1 RF+, $5 \mathrm{RF}-$ ). PMR patients consisted of 12 men and 12 women. All of them were ACPA-/RF- and did not meet 2010 RA criteria. Twenty patients met 2012 PMR classification criteria, and 4 patients without bilateral shoulder pain met Bird's criteria. Clinical features and statistical results are shown in the Table. Sixty-seven percent of RA patients and $13 \%$ of PMR patients had left-right differences in joint pain.

Scoring was performed based on clinical findings. Tenderness and/or swelling joint counts among wrists, fingers, ankles, and knees = each 1 point, left-right difference $=1$ point, no bilateral shoulder pain $=1$ point, no girdle pain $=1$ point, no fever $=1$ point; the maximum score was 8 . The mean score in RA patients was $4.8(\mathrm{SD}=1.44)$, whereas that in PMR patients was significantly lower at $1.5(0.98)$ $(P<0.001)$. Receiver operating characteristic $(\mathrm{ROC})$ curve analysis was used to determine the most suitable cut-off level to find RA. A score over 3 was $100 \%$ sensitivity and $87.5 \%$ specificity. All 6 ACPA-negative RA patients showed a score over 4 .

Table Clinical features in patients with rheumatoid arthritis (RA) and polymyalgia rheumatica (PMR)

\begin{tabular}{lllccc} 
& & & $R A \geqq 75(n=21)$ & $P M R \geqq 75(n=24)$ & $P$-value \\
\hline Age at onset (years) & & mean (SD) & $81.5(4.18)$ & $80.5(3.95)$ & 0.4 \\
Male & & $n(\%)$ & $6(33)$ & $12(50)$ & 0.1 \\
Time from onset to start of treatment (months) & median (IQR) & $2.0(1.5)$ & $1.5(1.4)$ & 0.09 \\
Joint/muscle pain at onset & Bilateral shoulder & $n(\%)$ & $10(48)$ & $20(83)$ & 0.03 \\
& Girdle & $n(\%)$ & $0(0)$ & $12(50)$ & $<0.001$ \\
& Wrist & $n(\%)$ & $19(91)$ & $7(30)$ & $<0.001$ \\
& Finger & $n(\%)$ & $4(19)$ & $0(0)$ & 0.04 \\
& Foot & $n(\%)$ & $6(29)$ & $0(0)$ & 0.007 \\
Systemic symptoms & Knee & $n(\%)$ & $13(62)$ & $2(8)$ & $<0.001$ \\
& Fever & $n(\%)$ & $3(14)$ & $14(58)$ & 0.002 \\
Rheumatoid factor positive & Body weight loss & $n(\%)$ & $6(29)$ & $14(58)$ & 0.07 \\
Anti-CCP antibody positive & & $n(\%)$ & $12(57)$ & 0 & $<0.001$ \\
C-reactive protein (mg/dL) at onset & $n(\%)$ & $15(71)$ & 0 & $<0.001$ \\
Matrix metalloproteinase 3 (ng/mL) at onset & mean (SD) & $6.1(5.66)$ & $8.4(4.61)$ & 0.1 \\
\hline
\end{tabular}

$\mathrm{SD}$, standard deviation; IQR, interquantile range; $\mathrm{CCP}$, cyclic citrullinated peptide

Conclusions: Pease et al studied RA at onset 60 years and over and PMR, and reported that arthritis of wrists and fingers was suggestive of RA ${ }^{1}$. However, in our study, small joint swelling was rare in RA patients 75 years and older. The scoring system we made might be useful for the differential diagnosis of ACPA-negative RA and PMR in elderly patients 75 years and older.

References:

[1] Pease CT, et al. Diagnosing late onset rheumatoid arthritis, polymyalgia rheumatica, and temporal arteritis in patients presenting with polymyalgic symptoms. A prospective longterm evaluation. J Rheumatol 32: 1043-1046, 2005.

Disclosure of Interest: None declared

DOI: 10.1136/annrheumdis-2017-eular.2016

\section{AB0293 METABOLIC SYNDROME AND INSULIN RESISTANCE IN ADULT EGYPTIAN FEMALES WITH RHEUMATOID ARTHRITIS}

${ }_{\text {A.A.-R. Youssef }}{ }^{1,1}$, N.O. Neseem ${ }^{1}$, M.A.-B. Omar ${ }^{2}$, O.M. El-Siri ${ }^{1}$

${ }^{1}$ Rheumatology \& Rehabilitation; ${ }^{2}$ Clinical Pathology, Mansoura University, Mansoura, Egypt

Background: Patients with Rheumatoid Arthritis (RA) have an increased risk for cardiovascular disease (CVD) due to higher prevalence of traditional risk factors (1), Insulin resistance (IR) is implicated in inflammatory diseases such as RA (2). The prevalence of Met.Syn and IR in Egyptian female RA patients has not been studied before.

Objectives: To find out the prevalence of Met.Syn and IR in a cohort of Egyptian females with RA and in controls and to study the associated risk factors Methods: 60 female RA patients and 30 healthy females matched for age were included according to the ACR/EULAR 2010 classification criteria. Disease activity was assessed using DAS- 28. IR using HOMA- index (HOMA- IR) (3) Met.Syn was defined according to the updated third report of the National Cholesterol Education Program's Adult Treatment Panel (NCEP-ATP III) criteria (4).

Results: Prevalence of Met.Syn in female RA patients is significantly (sig.) higher $(56.7 \%)$ than that of controls $(33.3 \%, P=0.04)$. IR is prevalent in $R A$ patients $(63.4 \%)$, Patients with Met.Syn exhibited sig. higher serum levels of TG $(P<0.001$,$) FBG (P=0.02)$, CRP $(P=0.02)$, Fasting insulin $(P=0.01)$ and IR
$(\mathrm{P}=0.03)$ than those without. Median CRP (24) and mean DAS- 28 (5.6 \pm 1.5$)$ in RA patients with increased IR are sig. higher than those of RA Patients with normal IR $(6.5, P<0.01) \&(4.7 \pm 1.5 P<0.04)$ respectively. Significant positive correlation was found between DAS-28 and IR ( $R s=0.3, P=0.03$ ). Using logistic regression, high systolic blood presser ( $\mathrm{OR}=1.2,95 \% \mathrm{Cl}: 1.02-1.39, \mathrm{P}=0.03)$ and elevated $\mathrm{CRP}(\mathrm{OR}=1.07,95 \% \mathrm{Cl}: 1.01-1.14, \mathrm{P}=0.04)$ have shown to be the significant independent predictors for the development of Met.Syn

Conclusions: Met.Syn. and IR are prevalent in female Egyptian RA patients adding to the $\mathrm{CV}$ risk of the disease and both are related to increased disease activity. Rheumatologists should pay an attention to control RA disease activity in addition to screen patients for components of the Met.Syn and introduce appropriate treatment strategies. Further studies are warranted to get more conclusive results.

\section{References:}

[1] Gremese, E., \& Ferraccioli, G. (2011). The metabolic syndrome: the crossroads between rheumatoid arthritis and cardiovascular risk. Autoimmunity Reviews, 10(10), 582-589.

[2] Goodwin, P. J., Ennis, M., Bahl, M., Fantus, I. G., Pritchard, K. I., Trudeau, M. E., ... \& Hood, N. (2009). High insulin levels in newly diagnosed breast cancer patients reflect underlying insulin resistance and are associated with components of the insulin resistance syndrome. Breast Cancer Research and Treatment, 114(3), 517-525

[3] Reilly, M. P., Wolfe, M. L., Rhodes, T., Girman, C., Mehta, N., \& Rader, D. J. (2004). Measures of insulin resistance add incremental value to the clinical diagnosis of metabolic syndrome in association with coronary atherosclerosis. Circulation, 110(7), 803-809.

[4] Grundy, S. M., Cleeman, J. I., Daniels, S. R., Donato, K. A., Eckel, R. H., Franklin, B. A., ... \& Spertus, J. A. (2005). Diagnosis and management of the metabolic syndrome an American Heart Association/National Heart, Lung, and Blood Institute scientific statement. Circulation, 112(17), 2735-2752.

Acknowledgements: We acknowledge the efforts received by all staff members of departments of Rheumatology and clinical pathology at Mansoura University Hospital, Egypt.

Disclosure of Interest: None declared

DOI: 10.1136/annrheumdis-2017-eular.1320

\section{AB0294 PREGNANCY IN RHEUMATOID ARTHRITIS - A ROMANIAN COHORT}

A. Bobirca ${ }^{1}$, F. Bobirca ${ }^{2}$, I. Ancuta ${ }^{1}$, C. Mihai ${ }^{1}$, C. Tataru ${ }^{1}$, C. Comsa ${ }^{1}$, M. Bojinca ${ }^{1}$, M. Micu ${ }^{3}$, A. Musetescu ${ }^{4}$, C. Ancuta ${ }^{5}$, V. Stoica ${ }^{1} .{ }^{1}$ Internal Medicine and Rheumatology; ${ }^{2}$ General Surgery, Cantacuzino Hospital, Carol Davila University of Medicine and Pharmacy, Bucharest; ${ }^{3}$ Division of Rheumatology, Department of Rehabilitation II, Clinical Rehabilitation Hospital, Cluj-Napoca; ${ }^{4}$ Rheumatology Department, University of Medicine and Pharmacy, Craiova; ${ }^{5}$ Clinical Hospital of Rehabilitation, "Grigore T. Popa" University of Medicine and Pharmacy, lasi, Romania

Background: Planning a pregnancy in rheumatoid arthritis (RA) meets several issues, mostly concerning potential drug toxicity and disease flares

Objectives: The purpose of this study is to evaluate pregnancy planning, RA activity during pregnancy and postpartum, pregnancy and fetal outcomes in a Romanian cohort of female patients diagnosed with RA.

Methods: This is an observational, ambispective study, including 58 RA Caucasian females with obstetric history after the onset of RA ( 20 females - prospective, 38 - retrospective). The cases were obtained from several Clinics of Rheumatology from Romania

Results: The mean age at inclusion was 37.1 years, age at RA diagnosis 3.9 years and mean age at conception 32.2 years. We recorded a total number of 96 pregnancies: 48 deliveries at term, 4 premature births, 15 elective abortions, 24 spontaneous abortions, and 5 ongoing. 34/96 (35.4\%) had at least one unplanned pregnancy, while being on treatment.

Concerning the exposure to synthetic DMARDs during the pregnancy: 6 patients received Leflunomide and 4 received Methotrexate during the first trimester, the pregnancy outcomes being: 3 spontaneous and 3 elective abortions, 3 normal birth (1-Cholestiramine wash-out), 1 premature twin pregnancy.

Regarding biologic DMARDs: 5 were exposed to Etanercept - 3 less than 3 weeks, ( 2 normal births and one elective abortion- due to Methotrexate use), 2 treated in second trimester: 1 only in the 15th and 16th weeks due to relapse normal birth, and the other one until week 20, pregnancy still ongoing.

One patient was treated with Certolizumab until week 12, the pregnancy is ongoing, and one with Adalimumab until week 4 , the fetus had intrauterine growth restriction, premature birth.

6 patients treated with Rituximab were included, last infusions were: 4 weeks before conception (1- spontaneous abortion and 1 normal birth), 48 weeks (2 normal births and 1 premature), and one at 4 weeks after conception - normal birth.

In 4 cases the patients stopped the biological DMARD before conception: Etanercept 6 months and 2 years, Adalimu-mab, 6 months with normal outcome, and for Tocilizumab 9 months (growth restriction)

$81.25 \%$ of our patients were in Remission or Low Disease Activity (by DAS28CRP) at conception and generally this status was maintained, excepting several situations. 
The average pregnancy length was 36.36 weeks and the mean birthweight was 2878.90 grams.2 growth restriction was identified and several atopic dermatitis, no teratogenic effect.

13 patients never had a new flare postpartum and in the others the mean time of postpartum flare was 12.1 weeks

Conclusions: Patients with RA can have successful pregnancies. More than $60 \%$ of pregnancies have been planned. Pregnancy decreases disease activity, but many deliveries are followed by RA flares. No fetal abnormalities were diagnosed. References:

[1] Østensen $M$, et all. State of the art: Reproduction and pregnancy in rheumatic diseases: Autoimmun Rev. 2015 May;14(5):376-86. doi: 10.1016/ j.autrev.2014.12.011. Epub 2014 Dec 30.

Disclosure of Interest: None declared

DOI: 10.1136/annrheumdis-2017-eular.6605

\section{AB0295 THE RECALL SURVEY: DATA FROM A MULTICENTER EDUCATIONAL EVENT ON PATIENTS WITH RA}

A. Delle Sedie ${ }^{1}$, E. Filippucci ${ }^{2}$, O. Epis ${ }^{3}$, P. Macchioni ${ }^{4}$, S. Tropea ${ }^{5}$, C. Bonali ${ }^{6}$ M. Canzoni ${ }^{7}$, A. lagnocco ${ }^{8} .{ }^{1}$ Rheumatology Unit, University of Pisa, PISA;

${ }^{2}$ Rheumatology Department, Università Politecnica delle Marche, Jesi;

${ }^{3}$ Rheumatology Unit, Ospedale Niguarda Milano, Milano; ${ }^{4}$ Rheumatology Unit, Reggio Emilia Hospital, Reggio Emilia; ${ }^{5} 5$ ASP7 Busacca Hospital, Ragusa;

${ }^{6}$ Rheumatology Unit, Ospedale San Paolo, Bari; ${ }^{7}$ Local Health Unit (ASL) Rome 1, Rome; ${ }^{8}$ Academic Rheumatology Unit, Università degli Studi di Torino, Torino, Italy

Background: Ultrasound (US) is able to show subclinical synovitis in patients (pts) with rheumatoid arthritis (RA) who are in clinical remission (CR); this has been proposed as a predictive factor for both clinical flares and progression of the structural damage in CR pts.

Objectives: To investigate the US characteristics of RA pts presenting either CR or low disease activity (LDA)

Methods: In 2015 an educational event focused on the added value of US in RA pts was held in 22 rheumatology centers in Italy. After a brief presentation on the evidence of US added value for the clinician given by expert sonographers (rheumatologists with a special interest in US that were performing US as their usual activity for many years), in every center, the local rheumatologists provided RA pts to be examined by US. All the US machines were identical both for type (Logiq E R7, General Electrics, with a 4.2-13 MHz linear probe) and settings (both for grey-scale and power Doppler (PD)). Pts signed an informed consent and a brief history of them was collected by the local rheumatologists (previous and current therapy, DAS28, HAQ score). The US examination was performed bilaterally on wrists, MCP and MTP joints, looking for synovitis (effusion, synovial proliferation and PD signal) and bone erosions. The positive findings were scored according to a 0-3 score for synovitis components and presence/absence for erosions; the number and size of the largest erosion was also registered.

Results: Demographic and descriptive data of the 433 pts examined are reported in Table I. Pts were divided on the basis of the DAS28 result. Statistically significant differences in age and disease duration were registered between the group in CR and the group with DAS28 $\geq 3.2(p=0,019$ and $p=0,012$, respectively), while no differences were found for HAQ or MTX use. Higher positivity of findings, regardless which was the score, was present in LDA group for effusion, synovial proliferation and PD signal. Significant differences were seen between $C R$ and LDA group for effusion (general, MCP and MTP joints; $p=0,011, p=0,026, p=0,017$ and $p=0,013$, respectively), PD positivity (general, wrist, MCP and MTP joints; $p=0,006, p=0,023$ and $p=0,05$, respectively) and erosions $(p=0,002)$, with higher positivity for pts in LDA.

Table 1. demographic data

\begin{tabular}{lccc}
\hline & DAS28 $<2.6$ & DAS28 2.6-3.2 & DAS28 $\geq 3.2$ \\
\hline $\mathrm{N}(\mathrm{M}: \mathrm{F})$ & $155(34: 121)$ & $170(39: 131)$ & $108(20: 88)$ \\
Age (mean $\pm S D ; y y)$ & $56.1 \pm 14.3$ & $59.2 \pm 12.2$ & $60.5 \pm 11.4$ \\
Disease duration (mean $\pm S D ;$ dd) & $2368.49 \pm 2565.39$ & $3274.16 \pm 2948.36$ & $1966.53 \pm 1884.58$ \\
\hline
\end{tabular}

Conclusions: The US assessment in this large cohort of RA pts showed frequent positive findings both in the CR and in the LDA group, confirming the results from previous studies. US is a useful imaging tool for the detection of subclinical joint abnormalities in RA.

Disclosure of Interest: None declared

DOI: 10.1136/annrheumdis-2017-eular.4475

\section{AB0296 HMG-COA REDUCTASE INHIBITOR DRUGS (STATINS) BENEFIT IN CARDIOVASCULAR RISK PREVENTION AND AMELIORATE DISEASE ACTIVITY IN PATIENTS WITH RHEUMATOID ARTHRITIS}

A. Hon, M. Sultanova. Cardiorheumatology, Tashkent Medical Academy, Tashkent, Uzbekistan

Background: Patients with active rheumatoid arthritis (RA) are frequently diagnosed with an atherogenic lipid profile and supra-aortic vessel ultrasound signs of atheromatous plaques, which have been linked to the inflammatory activity of RA. Recent experimental and clinical evidences suggest that the beneficial effects of statins are pleiotropic and have been proposed to have anti-inflammatory and immunomodulatory effects, inhibiting proinflammatory cytokines (IL-1 $\beta, 6,8$, TNF- $\alpha$ ), adhesion molecules (sICAM 1, E-, P-selectin), osteoprotegerin, which are implicated in RA pathogenesis. However, the beneficial role of statin therapy in clinical improvement and their benefit-risk profile are still debatable.

Objectives: To evaluate the effect of medium-term rosuvastatin therapy on lipid profile, endothelial dysfunction and RA activity in patients with rheumatoid arthritis (RA) in comparison with conventional disease modifying antirheumatic drugs (DMARD) therapy.

Methods: The study included 82 patients in the Specialized Course Outpatient Therapy Department of the 1st Clinic of Tashkent Medical Academy of age group between 44 and 65 years (mean $52 \pm 8.4$ ), predominantly female gender $(n=57,69.5 \%)$, with early RA (mean disease duration 9.2 \pm 2.4 months), and divided into 2 groups. Group $1(n=40)$ received methotrexate (MTX; 7.5 $\mathrm{mg} /$ week; plus prednisolone (10 $\mathrm{mg} /$ day). Group $2(\mathrm{n}=42)$ received MTX and prednisolone with the same previous doses plus rosuvastatin $(40 \mathrm{mg} /$ day). Lipid profile assessment comprised triglycerides, total cholesterol (TC), high-density lipoprotein cholesterol (HDL-C), low-density lipoprotein cholesterol (LDL-C). Disease activity was assessed by the disease activity score of 28 joints (DAS28), erythrocyte sedimentation rate (ESR), C-reactive protein (CRP) and visual analog scale (VAS). Disease activity, lipid profile and intima-media thickness (IMT) of common carotid arteries were measured before and after 85 days ( 6 months) of treatment.

Results: 4 patients receiving rosuvastatin were excluded due to abnormal liver function test parameters (De Ritis ratio $<0.9$ ), further assessment was thus performed on 78 (Group 2, $n=38$ ) early RA patients. Overall ESR (Group 1: 24.4 \pm 7.26 ; Group 2: $37.4 \pm 12.3$ ) and CRP (Group 1: $5.56 \pm 0.58$; Group 2: $3.71 \pm 1.23$ ) declined significantly during the treatment. The mean DAS28, unconditionally considered as the most important index of clinical disease activity in RA, was found to be lower $(p<0.05)$ in the adjunct statin-treated group (Group 2: $3.68 \pm 0.77$ ) than that of the conventional DMARD treated group (Group 1: $4.45 \pm 1.08)$. Statin significantly reduced LDL-C $(3.9 \pm 1.2 \mathrm{mmol} / \mathrm{l}$ to $3.3 \pm 0.8 \mathrm{mmol} / \mathrm{l}$; $\mathrm{p}=0.08)$ and increased HDL-C $(1.3 \pm 0.6 \mathrm{mmol} / \mathrm{l}$ to $2.0 \pm 0.4 \mathrm{mmol} / \mathrm{l} ; \mathrm{p}=0.06)$ after 6 months of treatment. However, rosuvastatin therapy showed no significant improvement in VAS score $(6.7 \pm 1.5$ to $6.9 \pm 0.6 ; p=0.41)$ and IMT $(1.04 \pm 0.09$ to $1.08 \pm 0.07 ; p=0.05)$.

Conclusions: Statins ameliorate RA activity, reduce potential cardiovascular risk in the context of atherosclerosis and mediate clinically apparent anti-inflammatory effects, but long-term effects and benefit-risk profile should be addressed in the management of elevated risk of cardiovascular events in RA patients.

References:

[1] Husain K., Hernandez W., Ansari R. A., Ferder L. Inflammation, oxidative stress and renin angiotensin system in atherosclerosis. World Journal of Biological Chemistry. 2015;6(3):209-217.

Disclosure of Interest: None declared

DOI: 10.1136/annrheumdis-2017-eular.6577

\section{AB0297 TREATMENT WITH LOW-DOSE PREDNISONE DOES NOT INFLUENCE BODY COMPOSITION DURING THE FIRST YEAR OF RHEUMATOID ARTHRITIS- A PILOT STUDY}

A. Raczkiewicz, O. Bujakowska, A. Juszkiewicz, B. Kisiel, J. Kur-Zalewska M. Tłustochowicz, W. Tłustochowicz. Internal Medicine and Rheumatology, Military Institute of Medicine, Warsaw, Warsaw, Poland

Background: Early change in body composition is one of the risk factors of CVD in rheumatoid arthritis (RA). These changes have been atributed to inflammation and decreased physical activity. Glucocorticosteroids (GCS) reduces inflammation but may influence body composition. There is limited information about the effects of low-dose GCS on body composition in early RA.

Objectives: The aim of the study was to assess the presence of altered body composition in patients with early rheumatoid arthritis (eRA) and to determine whether low-dose prednisone affects body composition.

Methods: 65 consecutive eRA patients (49 women) aged $61 \pm 14$ years were assessed at the time of diagnosis and after 12 months of treatment. All the patients had been treated with methotrexate (target dose $25-30 \mathrm{mg} /$ week) and tapered doses of prednisone (mean dose $4.8 \pm 3.4 \mathrm{mg} /$ day). Disease activity score (DAS28), Health Assesment Questionnaire (HAQ), body mass index (BMI), waist/hip ratio, comorbidities, physical activity and smoking were recorded. Total and regional lean mass and fat mass were measured with dual energy X-ray absorptiometry (DXA). DXA measures were repeated after 12 months in 34 patients ( 24 women) and compared with baseline data.

Results: At baseline fat free mass index (FFMl; $\mathrm{kg} / \mathrm{m}^{2}$ ) was below the 10 th percentile of a reference population in $32 \%$ of the women and $25 \%$ of the men. Reduced FFMI correlated with baseline ESR. Fat mass index (FMI) was above 90 th percentile in $28 \%$ of the women and $42 \%$ of the men. Fat mass index (FMl; $\mathrm{kg} / \mathrm{m}^{2}$ ) correlated with $\mathrm{HAQ}$, age and femoral BMD in women. After 12 months FFMI and FMI did not change significantly. There was no correlation between prednisone doses and the duration of prednisone treatment and changes in regional lean mass and fat mass. The decrase of FFMI (in 16 patients) was associated with higher mean ESR and lower vitamin D3 serum concentration. Conclusions: Low FFMI was common in patients with eRA. The treatment with 